\title{
DE LA LAGUNA AL OCÉANO : LA PRESENCIA VENECIANA EN EL GOLFO DE CÁDIZ (SIGLO XV) ${ }^{1}$
}

\section{From the Lagoon to the Ocean: Venetian Presence IN THE GULF OF CADIZ (FIfTEENTH CENTURY)}

\author{
RAUL GONZÁLEZ ARÉVALO \\ rgonzalezarevalo@ugr.es
}

UNIVERSIDAD DE GRANADA ${ }^{2}$

http://dx.doi.org/10.25267/Riparia sup.2019.i2.05

\section{RESUMEN}

Las investigaciones sobre la presencia veneciana en el Golfo de Cádiz son muy escasas. Este estudio busca ampliar el marco de estudio con la consulta de fuentes en gran medida inéditas, que han sacado a la luz nuevos datos sobre el conocimiento del territorio y su papel en la navegación y el comercio venecianos.

Palabras Claves: Toponimia, Historia y Arqueología del Reino de Granada

1 Este trabajo forma parte del Proyecto I+D «Las ciudades de la Corona de Castilla. Dinámicas y proyección de los sistemas urbanos entre 1300 y 1600» (HAR2017-82983P), financiado por el Ministerio de Economía y Competitividad y dirigido por los profesores María Asenjo González y David Alonso García.

2 Profesor Titular de Universidad. Historia Medieval. Departamento de Historia Medieval y Ciencias y Técnicas Historiográficas. Facultad de Filosofía y Letras, Calle Profesor Clavera s/n, Campus Universitario de La Cartuja, Granada.

R. González Arévalo, «De la laguna al océano: la presencia veneciana en el Golfo de Cádiz (siglo XV)» Suplemento RIPARIA 2 (2019), 113-141. 


\section{Abstract}

Studies on Venetian presence in the Gulf of Cadiz are scarce. The present paper aims to broaden the frame tackling with sources largely unheard of until very recently, which have provided with new data on the knowledge of the territory and its role in Venetian trade and navigation.

KEY WORDs: Cadiz, Sanlucar de Barrameda, Venice, historical geography, trade and navigation. 
Los estudios sobre la presencia veneciana en Andalucía son muy reducidos. La escasez y la dispersión de datos ha hecho que, a día de hoy, apenas contemos con publicaciones específicas $^{3}$. En consecuencia, no sorprende que se haya concluido razonablemente que apenas había súbditos de la Serenísima en el territorio, que no haya casi datos sobre su avecindamiento y son episódicos los que revelan la naturalización como castellanos ${ }^{4}$.

Centrándonos en el Golfo de Cádiz, las investigaciones se han ocupado fundamentalmente en el paso de las famosas mude de galeras mercantiles amparadas por el Estado, con escalas programadas en Cádiz y Sanlúcar de Barrameda ${ }^{5}$. Por su parte, las noticias sobre la existencia de un consulado conjunto con los florentinos en el puerto de Cádiz en las décadas centrales del siglo

3 Así lo confirma el estudio historiográfico que llevé a cabo en 2013, en el que apenas constataba media docena de publicaciones específicas sobre los venecianos, la mayoría centradas en el paso de las mude de Poniente y Berbería por las costas ibéricas. R. GonZÁlez ArÉvalo, Presencia diferencial italiana en el sur de la Península Ibérica en la Baja Edad Media. Estado de la cuestión y propuestas de investigación, Medievalismo, 22, 2013, 175-208, en concreto 188. Los estudios específicos a los que aludo son los siguientes: L. D'ARIENzo, La presenza dei veneziani in Andalusia all'epoca di Cristoforo Colombo", en Atti del Convegno "Il letterato tramiti e realtà del Nuovo Mondo: Veneria, il mondo iberico e l'Italia", Roma 1994, 203-230; reeditado en La presenza italiana in Spagna al tempo di Colombo, Roma 2010, 223-256, edición que sigo; más recientemente pueden verse mis trabajos: R. GonzÁLEZ Arévalo, Del Guadalquivir al Nilo. El testamento de Andrea de Razi (1477) y la comunidad veneciana de Sevilla a finales del siglo XV, Nuova Rivista Storica, en prensa; R. GonZÁlez Arévalo, Del Adriático al Atlántico: Venecia y Cádiz entre navegación, diplomacia y comercio (siglos XIV-XV), en prensa; R. GonZÁleZ ARÉVALO, Cum necessarium sit babere consulem nostrum in Sibilia. El consulado veneciano de Sevilla (1402-1407), en prensa.

${ }^{4}$ R. GonZÁlez ArÉvalo, Vecinos y propietarios: La integración de los italianos en las sociedades portuarias andaluzas (siglos XIII-XV), en J. Á. Solórzano TelecheA, B. ArízAga Bolumburu y M. BochaCA (eds.), Las sociedades portuarias de la Europa atlántica en la Edad Media, Logroño 2016, 249-284; isdem, Integración y movilidad social de los italianos en la Corona de Castilla: genoveses, florentinos y venecianos en la Andalucía bajomedieval, L. TANZINI y S. TOGNETTI (eds.), Competenze, conoscenze e mobilità sociale nell'Italia del basso Medioevo, Roma 2016, 375-401.

5 J.E. López de Coca CASTAÑer, Las galeras venecianas de Poniente y Berbería desde la perspectiva española, Medievalismo 16, 2006, 113-172. 
XV parecen responder a la importancia de la escala en la ruta de Poniente que en la existencia de una comunidad estable en el puerto ${ }^{6}$.

El presente texto se propone abordar la presencia veneciana en el Golfo de Cádiz de modo integrador, abordando el territorio como una unidad histórica, de la misma manera que se ha realizado hace poco al otro lado del Estrecho de Gibraltar, en el mar de Alborán ${ }^{7}$. Para ello se abordarán diversos aspectos claramente diferenciables y estrechamente relacionados entre sí: en primer lugar, el conocimiento del territorio, centrado en las informaciones sobre las condiciones de navegación y los puertos; a continuación, la estrategia institucional de la república, articulada mediante la diplomacia y la acción consular; posteriormente, la navegación de la flota veneciana, tanto de las galeras de la república como de las embarcaciones privadas; por último las informaciones sobre el comercio recogidas en fuentes específicamente venecianas.

\section{La raxion de'marineri: el conocimiento del territorio}

Más allá del debate sobre la realidad de su uso a bordo de las embarcaciones, no cabe duda de que portulanos y cartas náuticas desempeñaron un papel fundamental en el conocimiento del territorio para los extranjeros ${ }^{8}$. En este marco las

${ }^{6}$ R. GonzÁlez ArÉvalo, Florentinos entre Cádiz y Sevilla en los siglos XIV y XV, en E. Aznar Vallejo, R. J. González Zalacaín (coords.): De mar a mar. Los puertos castellanos en la Edad Media, La Laguna 2015, 273-308; isdem, En el Mediterráneo atlántico: Andalucia y las marinas italianas en la ruta de Poniente, en prensa.

7 R. GonZÁlez ArÉvalo, Presencias, interacciones y mutaciones italianas en el mar de Alborán (siglos XII-XV). Algunas reflexiones desde la historiografía, en B. Sarr (ed.), Alborán. Poblamiento e intercambios en las zonas costeras de al-Andalus y el Magreb, Granada 2018, 361-410.

${ }^{8}$ R. GonZÁlez ArÉvalo, La costa del Reino de Sevilla en la documentación náutica italiana (siglo XV), en A. Malpica Cuello, R. G. Peinado Santaella, A. FÁbregas García (eds.), Historia de Andalucía. VII Coloquio, Granada, 2010, CD-ROM, 301-317; V. Muñoz Gómez, Para el conocimiento de la costa de la Andalucía atlántica (siglos XIV-XVI): descripciones, relaciones y documentación náutica, Historia. Instituciones.

«De la laguna al océano: la presencia veneciana en el Golfo de Cádiz...» 
informaciones de los textos venecianos son altamente valiosas. El primero de ellos es el manuscrito de Michele da Rodi (1434-36), que contiene un Portolan de Spagna e le so traverse, del que, además, excepcionalmente, se conoce el autor: "Portolan fatto per Zuan Pires, pedotta del mar di Fiandra". Es decir, se trata de uno de los tantos pilotos castellanos, Juan Pérez, enrolados para afrontar la travesía del Atlántico desde Cádiz hasta Flandes?:

Trafigar e Mazamoias, sirocho maistro, tuo' in puocho de la tramontana, lege 7.

Trafigal e pontal de Sancta Maria, levante e sirocho, lege 3.

Chavo San Vizenzo e Tazeri, levante sirocho, lege 12.

Salmerina chon pontal de Sancta Maria, ponente levante, lege 25.

Pontal de Sancta Maria e Porchas de Chades, zoè li schogi, quarta de levante sirocho, lege 28.

Ponctal de Sancta Maria, Arenas Gurdas de Sivillia, quarta de levante al griego, lege 19.

Además, en el apartado Aque e marie de Spagna señala que "L'aqua de Chades e de Farnon e de Lisbuna quando la luna in griego e tramontana, piena mare. In Barameda la luna quarta de sirocho alo levante, basa" ${ }^{10}$. Es decir, se recogen datos relativos a las peligrosas secas, los bancos de arena en los que podían encallar las embarcaciones; pero también sobre las condiciones de bajamar y pleamar y las corrientes. De hecho, el texto abre con la descripción entre el

Documentos 40, 2013, 179-205; isdem, Puertos, abras, cabos e islas: la topografía medieval de la costa atlántica de Andalucía a través de las cartas portulanas (ss. XIVXVI), en E. Aznar VAllejo, R. J. GonzÁlez Zalacaín (coords.): De mar a mar. Los puertos castellanos en la Edad Media, La Laguna 2015, 179-211; isdem, Derroteros, memoriales y saberes náuticos sobre las costas meridionales ibéricas durante la Era de los Descubrimientos (1526-c. 1555), Chronica Nova 42, 2016, 425-451.

9 P. O. Long, D. McGee y A. M. STAhl (eds.), The Book of Michael of Rhodes. A FifteenthCentury Maritime Manuscript. Volume 2: Transcription and Translation, edited by Alan M. Stabl, Cambridge (Mass.) 2009, 350. Según Piero Falchetta en la distancia entre el cabo de Trafalgar y la punta de Santa María hay un lapsus calami, pues la distancia son 33 leguas, y no 3 leguas. P. FalchetTA, The Portolan of Michael of Rhodes, en P. O. LONG, D. MCGEE y A. M. STAHL (eds.), The Book of Michael of Rhodes..., vol. 3, 198.

10 Ibidem, 364. 
cabo de Trafalgar y el cabo de San Vicente, con informaciones sobre Trafalgar, El Puerto de Santa María, la Roca Salmedina, las Puercas de Cádiz, Sanlúcar de Barrameda y Arenas Gordas.

La comparación con la información recogida en el célebre manual hasta hace poco atribuido a Piero de' Versi, Raxion de' marineri (1444), del que se ha descubierto que fue autor en parte el propio Michele da Rodi, explica que el Portolan fatto per tuto el mar de Spagna incluido se basa directamente en el texto original de Juan Pérez. De la misma manera, la comparación entre los textos de Rodi y Versi con el anónimo Alcune raxion per marineri (1444/5) deja poco espacio para la duda, pues resulta evidente que es una variante del segundo allí donde coinciden ${ }^{11}$.

En los dos últimos textos, las dos variantes del manual de Piero de' Versi, se incluyen las informaciones de interés también para la navegación de la muda de Poniente, especificando "le spexe che se fa per le galie de Fiandra per le schale in ogni luogo":

A Chades l'armiario die' desmontar in tera per lo muodo avemo ditto indriedo e andar al nostro chonssolo e chon lui

\footnotetext{
11 Así en Versi: “Tranfiguar e Mazamoias se varda sirocho e maistro, ma tuo' un pocho de la tramontana e de l'ostro e sono lege 7.

Tranfalgar e pontal de Sancta Maria se varda levante e sirocho e ponente maistro lege 3.

Chavo de San Vizenzo e Tazeri se varda levante e sirocho ponente maistro lege 12.

Salmerina con pontal de Sancta Maria se varda ponente e levante lege 25.

Pontal de Sancta Maria e Porchas de Chades, zoè li schogi, se varda quarta de levante al gri(e)go e quarta de ponente al garbin lege 19.

[...] L'aqua de Chades e de Farnon e de Lisbona quando la Luna in griego e tramontana e ostro e garbin è piena mar.

In Baramede quando la luna quarta del sirocho a lo levante e quarta de maistro al ponente, bassa mar e quarta de garbin al ponente e quarta de griego a lo levante piena".

P. DE' Versi, Raxion de' marineri. Taccuino nautico del XV secolo, A. CONTERIO (ed.), Venecia 1991, 65 y 72. La misma información se repite, básicamente, en el anónimo Alcune raxion per marineri. Un manuale veneziano del secolo $X V$ per gente di mare, $\mathrm{O}$. PitTARello (ed.), Venecia 2006, 173-174 y 180. Sobre la autoría de Michele da Rodi del portulano posteriormente atribuido a Pietro de' Versi puede verse P. FALCHETTA, The Portolan..., 196-197.
}

«De la laguna al océano: la presencia veneciana en el Golfo de Cádiz...» 
die' andar là che fa luogo per domandar el salvochondutto e quello se tuo' per scritto.

Pagasse in lo ditto luogo per anchorazo per galia ducato 1 e per la galia di misier lo chapetagno paga ducati 2 e si per aventura andassi in primo a Tarifa e queli volesse esser pagadi, pageli e feve dar el boletin per non pagar un'altra volta a Chades.

Pagasse in lo ditto luogo per chonsolazio ducato un per galia.

Pagasse per el scrivan o noder che scriverà el salvochonduto de quela moneda mezo ducato.

Pagase de ritorno de le galie in deto luogo per anchorazo ducato $4^{12}$.

Más allá de los gastos por anclaje y salvoconducto que cobraban los castellanos, a los que se añaden el derecho de consulado y del escribano que dará fe del salvoconducto, es importante detenerse en la información sobre Tarifa, donde se podían abonar también las tasas de anclaje y salvoconducto, por lo que recibirían un boletín que justificaría el pago ante las autoridades gaditanas. Así se entiende también que ante el mal estado de la mar, las galeras florentinas de Poniente en octubre de 1429, al salir de Algeciras, pudieran refugiarse en Tarifa a la espera de mejores condiciones para continuar la ruta hasta el norte $^{13}$. Sin embargo, la parada florentina se debió a circunstancias excepcionales y no hay tampoco datos que confirmen que fuera una parada habitual para los venecianos, de modo que hay que concluir que el papel de Tarifa para las flotas

\footnotetext{
12 Pietro de' Versi, Raxion de' marineri..., 82. Una información similar, sin referencia al derecho de consulado, en Alcune raxion per marineri..., 147-148: "A Chades tti armiraio zontto al porto tti diè desmontar in tera et andar al nostro chonsollo et llui ve menerà là dove fa luogo per domandar el salvochodutto et quello se tuò per scritto; pagasse per chollui che schriverà, zoè el sanvochondutto, meza dobla. Pagasse per anchorazo in lo ditto luogo per gallia ducati 1 et per quella de misier lo chapetagno ducati 2; et sse per aventura arevassi avantti a Tarifa et quelli vollesse esser pagadi, pagè et feve dar un bollettin per non pagar un'altra volltta. Pagasse in lo ditto luogo per tutte ducati 4".

13 R. GonZÁlez ArÉvalo, Corso, comercio y navegación en el siglo XV: Castilla y las galeras mercantiles de Florencia, En la España Medieval 34, 2011, 68-69.
} 
italianas era absolutamente contingente y todo el protagonismo recaía en Cádiz.

Otro documento veneciano más contiene informaciones en el mismo sentido. Se trata de un Portolano del mare datado en 1477 y publicado por primera vez en Venecia en 1490 por Bernardo Rizzo, durante largo tiempo atribuido al célebre navegante y descubridor Alvise da $\mathrm{Ca}$ ' da Mosto. El texto contiene a su vez un Portolano del mar maggiore en el que se describen las costas gaditanas:

Dal capo S. Vincenzo insino alla bocca del fiume di Sibilia sono miglia 180. Entro levante e sirocco, dalla detta bocca alla cittade di Sibilia sono miglia 60. Ancora alla detta bocca al capo che vien detto Fermendina miglia 10. Per mezo verso libecchio e poi dalla detta bocca miglia 5 . Per libecchio è una secca che ha nome Pozaro, e è sopra acqua, e se vorrai in lo detto fiume intrare, guardati da una secca che vien detta Zizare. E verso ponente e levante è un'altra secca, che vien detta Cantara, e è diritta ad un capo che ha nome Sirocco, se vorrai intrare in lo detto fiume. Sirocco con nave in prima scandaglia in fondo, e metteti i segnali, anderai sicuro, la detta cittade Fermendina fu cittade, e quando vieni per intrare, honora la ponta della detta Fermendina, quando l'acqua n'è piena circa miglia 1, e dalla detta Fermendina all'isola di Cades sono miglia 20. Per sirocco alla detta isola è bon porto, che dreto ha una cittade, che è guastata simigliante alla prima verso tramontana, e la è fondo di passa 6 d'acqua, e dallo detto porto insino alla ponta della detta isola verso ponente per lo canal è fondo di passa 8 di acqua.

Dal detto Cades a Trafegar sono miglia 30 per sirocco, sopra il detto Trafegar in mar è una secca miglia 7 a garbin, e puossi andar dentro la secca e la terra, lungi dalla terra miglia 1 e mezzo; dallo detto Trafegar a Tarifa sono miglia 30 per sirocco verso levante un poco: dalla detta Tarifa al capo di Sazedara verso ponente miglia 10 dentro levante e sirocco. Dallo detto capo allisola di Cazerada miglia 5 per levante. In la detta isola 
mezzo è buon fondo, e buon porto a Barfazza di Villa. Ivi è fondo di passa 8 in capo della detta isola ${ }^{14}$.

El texto es el más detallado de todos los consultados hasta el momento. No solo da indicaciones precisas de hitos peligrosos como Salmedina -el arrecife frente a Chipiona en el acceso hacia la barra de Sanlúcar de Barrameda, tradicionalmente interpretada como ciudad sumergida bajo las aguas- o diversas secas en la desembocadura del Guadalquivir, sino que también contiene informaciones exactas sobre el puerto de Cádiz y las distancias sucesivas entre hitos sucesivos como la punta de Trafalgar, la punta del Carnero (capo di Sazedara) y, finalmente, Algeciras (Cazerada), aunque el nombre otorgado al primero induce a confusión por homonimia con el segundo, circunstancia también presente en el Portolano palatino conservado en Florencia, donde ambas reciben el nombre de chapo de Isalcadra e isola d'Isalcadra' ${ }^{15}$.

Con todo, para el tema que nos ocupa, interesa particularmente la descripción que se hace en el portulano de la isla y la bahía de Cádiz:

Chades è citade ed è ixola, e si è longa mia 30 e da tramontana de la cità à mia 8 è lo porto de Sancta Maria, ed è una bona vila e grande e de là se va suxo per aqua fin a la cità de Seres e cum la marea granda entrano le nave nel dito porto. L'ixola de Chades volze intorno mia 60 ed è longa e streta e in tal logo non è 1 mio, e però lo canal è tra la terra ferma e l'ixola ed è bon sorzador, e a la bocha de levante è streto e seco e piere che foza un ponte roto, che antigamente se andava da la terra ferma a l'ixola, per mezo la cità, e a lo porto usado, dove vano le nave, si è bon fondi de passa 6 in 8 d'aqua e driedo la cità, de ver ponente, si è uno scoieto pericoloso et fano da ponente e

\footnotetext{
14 A. DA CA’ Da Mosto, Portolano del mare, Venecia 1883, edición que sigo. El original se conserva en la Biblioteca de San Marcos en Venecia.

15 R. GonZÁlez Arévalo, La costa..., 305.
} 
maistro una secha a nome i Porzi e tra maistro e tramontana a presso terra ferma è una altra secha a nome Sancta Croxe de Chades ${ }^{16}$.

La descripción de portulano anónimo es muy detallada en su extensión, superior a la de otros portulanos conservados en Florencia. Además de recoger la distancia con El Puerto de Santa María, destaca la información del acceso por agua hasta Jerez de la Frontera con la marea alta, que se realizaba a través de las barcas del Guadalete ${ }^{17}$. Respecto a la propia isla de Cádiz, además de proporcionar medidas que coinciden con otras de fuentes similares, destaca la referencia al Puente Zuazo -el acueducto de época romana que los repobladores cristianos confundieron con un puente de acceso a la ciudad-así como las secas de las Puercas de la Santa Cruz.

La fama del puerto era importantísima, como recordaba en 1519 el diario de un mercader milanés anónimo, cuando situaba a la ciudad como cabecera de un sistema portuario más amplio que en realidad englobaba también las localidades de Rota, El Puerto de Santa María y, después de su fundación en 1483, Puerto Real:

He Cadis un porto grandissimo et bonissimo, ove capitano navilij asayssimi, et he di questa forma [dibujo] et a l'intratta de l'una pontta a l'altra li he meza lega et non più, et circuisse circa a leghe 10, et su una delle ponte he Cadis, et su l'altra he uno loco chiamato Rotta, et quasi ad uno terzo da uno cantto he

\footnotetext{
16 A. DA Mosto, Il portolano attribuito ad Alvise da Ca' da Mosto, Bollettino della Societa Geografica Italiana 3 (VI), 1893, 554.

17 J. Abellán PÉrez, Las barcas del Guadalete: las relaciones entre la villa de El Puerto de Santa María y la ciudad de Jerez de la Frontera en época de los Reyes Católicos, Revista de Historia de El Puerto 47, 2011, 9-23.
}

«De la laguna al océano: la presencia veneciana en el Golfo de Cádiz...» 
Portto Reale, et quasi a l'altro terzo dall'altro cantto he il Portto Santa maria, loco de fochi $1000^{18}$.

Respecto a Sanlúcar de Barrameda, sorprende su ausencia en los manuales de marinería citados. Cabe recordar que se trataba de una escala de la muda de Berbería, instituida por la Serenísima en 1436. Sin embargo, en su diseño inicial el convoy de galeras finalizaba el viaje de ida en Cádiz. Efectivamente, Sanlúcar solo cobró importancia en las décadas centrales del siglo $\mathrm{XV}$, siendo incluida por primera vez en las órdenes oficiales de 1443, cuando figura como cabecera final de línea con una escala prevista de 24 días, y por última en las órdenes de 1462, cuando la parada prevista era de 20 días $^{19}$. En consecuencia, es lógico que los manuales venecianos de las décadas de 1430 y 1440 no la incluyeran entre sus informaciones.

Comoquiera que fuera, las informaciones posteriores no son mucho más prolijas. El diario de un mercader milanés anónimo anotaba hacia 1519 referente a la desembocadura del Guadalquivir que "va esso fiume in mare a Santo Lucaro, loco apresso a Cadix a leghe 5 et apresso a Sibilla a leghe 17, et la marea vene nel dicto fiume insino a Sibilla" 20 . Sin embargo, no cabe ninguna duda del papel de la capital ducal de los Guzmanes, como confirma claramente la primera versión del Portolano del Mediterraneo, documento del siglo XV custodiado en la Biblioteca Nacional Central de Florencia:

\footnotetext{
${ }^{18}$ L. Monga (ed.), Un mercante di Milano in Europa. Diario di viaggio del primo Cinquecento, Milán 1985, 140.

${ }^{19}$ El estudio más reciente de esta línea en relación a la Península Ibérica, incluyendo el papel de Sanlúcar de Barrameda, se ha abordado en R. GonZÁlez ArÉvalo, Acordes y desacuerdos. Navegación y comercio de las galeras mercantiles de Venecia y Florencia en el Mediterráneo ibérico desde una perspectiva comparada, en R. GonZÁLEZ ArÉvalo (ed.), Navegación institucional y navegación privada en el Mediterráneo medieval, Granada 2016, 145-191, y en concreto 167-171.

${ }^{20}$ L. Monga (ed.), Un mercante di Milano..., 136.
} 
bisognati piloto per entrare dentro e entra dritco infino a Borramida; achostati senpre a banda sinistra allo terreno per cierte basse che vi sono, e.nno entrare con montante. La chonoscença de lo rio di Sibilia siè uno chastello chi si chiama Sanlucha di Barramida, tre torri che vi sono ${ }^{21}$.

Nótese cómo frente a la denominación tradicional en la documentación italiana, Rio di Sibilia, la población viene citada por su nombre, Sanlúcar de Barrameda, en una distinción poco habitual en la documentación náutica de la época.

\section{Venecia, Cádiz y Sanlúcar de Barrameda en la diplomacia veneciana del siglo $\mathrm{XV}$}

Con el desarrollo de una comunidad estable en Andalucía en el siglo XV, inician las noticias sobre la apertura de consulados venecianos en Andalucía. El más conocido de todos es el de Sevilla. Efectivamente, en noviembre de 1398 Enrique III de Castilla confirmó el privilegio concedido anteriormente por su padre, Juan I, que incluía el derecho a abrir un consulado similar al de genoveses y catalanes en la capital hispalense. Desafortunadamente, queda mucho por averiguar de dicho consulado, del que apenas conocemos el nombre de tres titulares: micer Angelo Venerio (1403), Antonio da Ca da Mosto (1465) y Lorenzo Sacente $(1480)^{22}$.

Por su parte, todavía no se ha podido establecer en qué momento se decidió abrir un consulado en Cádiz. Las primeras noticias proceden de las "le spexe che se fa per le galie de Fiandra per le schale in ogni luogo" recogidas por Pietro de' Versi en su Raxion de' marineri de 1444, que especifica que el almirante de la muda de Flandes debía dirigirse al cónsul de la nación para abonar los

${ }^{21}$ R. GonZÁlez ArÉvalo, La costa..., 304-307.

22 Para el consulado sevillano véase R. GonZÁlez Arévalo, Cum necessarium..., en prensa.

«De la laguna al océano: la presencia veneciana en el Golfo de Cádiz...» 
derechos de anclaje y salvoconducto ${ }^{23}$. En 1459 hay noticias de un cierto Maryn de Grauel, que figura como cónsul de los venecianos trasladándose desde Cádiz a Jerez de la Frontera para tomar la vecindad ${ }^{24}$. El nombre, con toda probabilidad, refleja la versión castellanizada y distorsionada de Marino Crivelli, que figura asimismo como Marin de Creneli en el padrón de vecinos de Cádiz de 1467 como cabeza de una casa con trece personas, y en 1468 recibiendo como Marín de Crinile dos fanegas del abastecimiento de pan y trigo enviado desde Jerez de la Frontera $^{25}$. Por lo tanto, siguiendo una práctica habitual en el siglo XV, la función consular estaba encomendada a un ciudadano veneciano arraigado en el territorio. Hasta tal punto es así que incluso llegó a avecindarse en la vecina Jerez de la Frontera en 1459, si bien parece claro que regresó a Cádiz, como confirma el padrón de vecinos de 1467.

No conocemos el funcionamiento interno del consulado gaditano, pero sí que no atendía exclusivamente los intereses venecianos. Efectivamente, Marino Crivelli figura en 1462 como cónsul de los florentinos. En calidad de tal recibió del capitán Bongianni Gianfigliazzi los paños para uso personal y los libros de leyes que João Rodrigo, obispo de Coímbra, había embarcado en las galeras florentinas de Poniente de ese año ${ }^{26}$. En consonancia con los derechos pagados por la compañía Da Colle, que incluían los de consulado, hay que situar el gasto por "anchoraggio e salvocondotto e diritto di consolaggio a Cadice fiorini 10

\footnotetext{
23 Vid supra y nota 12.

24 "Otra tal vesindad tomó e fue reçebido por todos los dichos corregidor e regidores a Maryn de Grauel cónsul de los veneçianos, vecino de la dicha çibdad de Cadis. E dio por su fiador a Juan Sanches del Puerto, vecino desta çibdad el qual otorgó la dicha fiança en la manera sobredicha". Archivo Municipal de Jerez de la Frontera, Actas Capitulares, Año 1459, f. 4r, 17-I-1459. Agradezco al profesor Emilio Martín Gutiérrez que me facilitara el texto citado.

25 E. MARTín GutiÉrReZ, Nuevos datos sobre la población y los genoveses en la ciudad de Cádiz. Una relectura, En la España Medieval 29, 2006, 214, 216 y 219.

26 M. BERTI, Le aziende Da Colle: una finestra sulle relazioni commerciali tra la Toscana ed il Portogallo a metà del Quattrocento, en Toscana e Portogallo, "Miscellanea storica nel $650^{\circ}$ anniversario dello Studio Generale di Pisa", Pisa 1994, 83 (nota 103).
} 
larghi" entre los registros de gastos de las galeras florentinas de Poniente que regresaron a Porto Pisano en $1467^{27}$. La identificación de Marino Crivelli como cónsul de los venecianos y de los florentinos me ha llevado a plantear que la institución gaditana era un consulado conjunto, al estilo de otros localizados en el Mediterráneo ${ }^{28}$. Comoquiera que fuera, las noticias sobre el consulado gaditano y el pago de derechos consulares en los registros florentinos todavía no se han visto confirmados en fuentes contables relacionadas con la navegación de las galeras venecianas $^{29}$.

La presencia de los cónsules en Cádiz estaba sujeta a las circunstancias de cada momento. Así, por ejemplo, en mayo de 1508 Francesco Cornaro, embajador veneciano en Castilla, informaba a la república de que el 7 de abril había enviado a Cádiz a su correo, Pacino, con los salvoconductos para las galeras de Flandes. Sin embargo, el emisario los entregó al cónsul el 21 de abril en las afueras de la ciudad, a cierta distancia, debido a la peste que la asolaba. De hecho, mientras duraba la epidemia el cónsul, cuyo nombre no se conoce, se había instalado en El Puerto de Santa María, donde esperaba que llegara la muda a lo largo del mes de mayo. En sus manos tenía aún los salvoconductos originales concedidos para el viaje de ida, que habían llegado después de que las galeras hubieran zarpado. Además, el correo informó al embajador de que había subido a bordo de la embarcación del corsario Chiara, desde donde observó una bandera con la efigie del dogo veneciano con una soga alrededor del cuello y grilletes en los pies, siendo arrastrada en el agua por la popa, como muestra de desprecio a la Señoría.

27 Archivio di Stato di Firenze, Consoli del Mare, VII, f. 71 r, 7-V-1467.

28 R. GonZÁlez ArÉvalo, Florentinos..., 303-306.

${ }^{29}$ L. Greco (ed.), Quaderno di bordo di Giovanni Manzini, prete-notaio e cancelliere (14711484), Venecia 1997; S. Montemezzo, Giovanni Foscari. Viaggi di Fiandra 1463-1464 e 1467-1468, Venecia 2012.

«De la laguna al océano: la presencia veneciana en el Golfo de Cádiz...» 
La afrenta mereció la protesta del embajador veneciano ante Fernando el Católico, que prometió enmendar la situación ${ }^{30}$.

El aspecto más sorprendente de los contactos diplomáticos entre Castilla y Venecia lo constituye a buen seguro la diplomacia privada desarrollada por los Guzmán, señores de Sanlúcar de Barrameda. El puerto sanluqueño, situado en la desembocadura del Guadalquivir, fue etapa fundamental de la muda de Berbería entre 1442 y 1462, a lo que no serían ajenos los privilegios concedidos por el I duque de Medina Sidonia, Juan Alonso Pérez de Guzmán, posteriormente renovados a perpetuidad en diciembre de 1468 por su hijo, Enrique de Guzmán ${ }^{31}$.

Es posible interpretar la renovación de los privilegios en sus dominios como un aspecto de la lucha con los Ponce de León por el dominio político y económico del Reino de Sevilla. En este sentido, cabe tener en cuenta que las galeras de Berbería, las únicas que recalaban en Sanlúcar, habían dejado de cruzar el Estrecho de Gibraltar después de 1462. Y de otra, hay que recordar que Juan Ponce de León, conde de Arcos y futuro marqués de Cádiz, se hizo con el señorío sobre la ciudad hacia 1467. La escala más importante de los venecianos en la Corona de Castilla reportaba importantes beneficios comerciales a la ciudad $^{32}$. En consecuencia, no puede ser casualidad que en 1468 el duque de Medina Sidonia escribiera a la Señoría ratificando los privilegios concedidos por su padre, en un intento por atraer de nuevo la presencia veneciana a sus dominios, tal vez en detrimento del papel de Cádiz y a favor de Sanlúcar de

30 Calendar of State Papers Relating To English Affairs in the Archives of Venice, Volume 2, 1509-1519, R. BROWN (ed.), Londres 1864, §1337, 579.

31 Libri commemoriali della Repubblica di Venezia. Regesti, Venecia 1901, tomo V, libro XVI; \$23, 3-XII-1468.

32 Una muestra significativa en M.Á. LADERO QUESADA, Unas cuentas en Cádiz (14851486), Cuadernos de Estudios Medievales II-III, 1974-75, 85-120. 
Barrameda. Sin embargo, el movimiento no surtió los efectos deseados, pues hay constancia de que los mercaderes venecianos siguieron negociando desde el puerto gaditano con los de El Puerto de Santa María, Sanlúcar de Barrameda, Jerez de la Frontera e incluso Sevilla ${ }^{33}$.

Por ende, parece evidente que los intentos de los Guzmán por asegurar la continuidad veneciana en su territorio no tuvieron mucho éxito, pero no cejaron en su intento. Más aún, una vez extinguido el señorío de los Ponce de León sobre Cádiz en 1493 y en competencia ahora con la propia Corona, en el contexto de los esfuerzos de los almojarifes de Sevilla por extender su jurisdicción a la costa andaluza, Juan Alonso Pérez de Guzmán, III duque de Medina Sidonia, escribió de nuevo al dogo veneciano el 24 de abril de 1499, solicitando esta vez la inclusión de Sanlúcar de Barrameda en el recorrido hacia el Mar del Norte. La respuesta no tardó en llegar: en agosto de ese mismo año Andrea Barbarigo le manifestaba que nada podía hacer ya para el viaje de ese año, cuyo encante había ocurrido meses antes y con las galeras prontas para zarpar, pero haría lo posible por satisfacer su petición para el viaje del año siguiente ${ }^{34}$. A pesar de las promesas, no hay noticias de que tampoco en el siglo XVI la muda de Flandes, activa hasta 1534, recalara en Sanlúcar de Barrameda, al contrario de lo que ocurre en Cádiz.

\footnotetext{
33 J.E. LÓPEZ DE CoCa CASTAÑer, Las galeras..., 157.

34 Archivo Ducal de Medina Sidonia, Fondo Medina Sidonia, leg. 2395, doc. 27, 9-VIII1499. Para la rivalidad de los Guzmanes con la Corona y los almojarifes pueden consultarse los trabajos de J.D. GONZÁlez ArCE, Pugnas entre monarquía y aristocracia por el control de los almojarifazgos costeros y otros derechos aduaneros de la Andalucía atlántica en el reino de los Reyes Católicos, Anuario de Estudios Medievales 47/1, 2017, 73-106, y La adaptación de la fiscalidad aduanera a los intereses repobladores, comerciales y políticos. Andalucía, 1241-1555, Hispania LXXVIII ( ${ }^{\circ}$ 258), 2018, 39-67.
}

«De la laguna al océano: la presencia veneciana en el Golfo de Cádiz...» 


\section{La navegación veneciana en el Golfo de Cádiz: galeras mercantiles y embarcaciones privadas}

El paso de las mude de Poniente y Berbería por las costas ibéricas ya ha sido abordado con detenimiento ${ }^{35}$. Sin embargo, es oportuno insistir en la ruta y las escalas efectuadas por los convoyes venecianos.

A pesar de que el manual de Pietro de' Versi señala la posibilidad de obtener el salvoconducto para el territorio castellano en Tarifa, las órdenes oficiales de la muda de Flandes establecía claramente que, una vez pasado el Estrecho de Gibraltar, las galeras debían dirigirse directamente a Cádiz, antes de continuar sin más escalas para Southampton, toda vez que la parada de Lisboa desapareció a principios del siglo XV. En ningún momento señala el manual de 1444 la posibilidad de detenerse en Sanlúcar de Barrameda, tanto menos de remontar el Guadalquivir hasta Sevilla, como afirmaba Doris Stöckly y dudó acertadamente José Enrique López de $\mathrm{Coca}^{36}$. Efectivamente, este último fue capaz de establecer que las galeras que se podían desviar hasta Sevilla eran las de Berbería ${ }^{37}$. En todo caso, también cabe especificar que las órdenes oficiales del Senado establecían como cabecera final de la línea Sanlúcar de Barrameda, nunca la capital hispalense.

Hay que tener presente que la escala de Sevilla no tenía sentido desde el punto de vista de la navegación, pues demoraba el ritmo de manera injustificada en la ruta de Poniente. Tampoco había una comunidad mercantil importante en la capital del Guadalquivir, y los mercaderes de la república obtenían buenos resultados en Cádiz, como se verá más adelante.

35 J.E. López de Coca Castañer, Las galeras...; R. GonzÁlez Arévalo, Acordes y desacuerdos...

36 D. STÖCKLY, Le système de l'Incanto des galées du marché à Venise, fin XIIIe-milieu XVe siècle, Boston-Leyden 1995, 159.

37 J.E. López de CoCa CASTAÑER, Las galeras..., 121-122 y 126-127. 
El número de galeras que componía el convoy fue variable. En el siglo XIV osciló entre cinco y dos, mientras que en la primera mitad del siglo XV se estabilizó en cuatro. También los días de escala variaron: las órdenes de 1336 establecían opcionales de dos a cuatro jornadas, mientras que hasta mediados del siglo XV parece que fue habitual que la parada durara cuatro o cinco días. La importancia de Cádiz en la navegación veneciana se ve confirmada porque las órdenes del Senado establecían que de regreso hacia la laguna el puerto gaditano y Mallorca fueran las únicas escalas contempladas ${ }^{38}$. En el siglo XVI los días previstos aumentaron a seis ${ }^{39}$.

Naturalmente, se trataba de un planteamiento ideal, pero no siempre encuentra correspondencia en otro tipo de documentación. Las escalas dependían de las contingencias propias de la navegación y del escenario político del momento. Así por ejemplo, la muda de Flandes que regresaba desde Inglaterra en 1477 se vio atrapada en el puerto de Cádiz por la flota francesa y no se aventuró a salir hasta que llegó una escolta desde Venecia para acompañarles de vuelta al Adriático ${ }^{40}$. Posteriormente, en el accidentado viaje de 1521-23, el capitán Vincenzo Priuli escribió al dogo veneciano desde Laredo en diciembre de 1521 para informar de que el mal estado de la mar obligó a prolongar la parada durante 27 días ${ }^{41}$.

Respecto a Sanlúcar de Barrameda, mientras la muda de Berbería cruzó el Estrecho de Gibraltar las órdenes oficiales del Senado decretaron que fuera la escala más extensa de la línea,

\footnotetext{
38 D. STÖCKLY, Le système..., 152-165.

39 Así lo decretó el Senado en el viaje de 1517. Calendar of State Papers..., vol. 2, Suplement II, 364.

40 A. RudDock, Italian merchants and shipping in Southampton, 1270-1600, Southampton 1951, 224.

41 F. Ortalli (ed.), Lettere di Vincenzo Priuli, capitano delle galee di Fiandra, al doge di Veneria, 1521-1523, Venecia 2005, cartas 5 y 6, pp. 5-6, 17 de septiembre y 13 de diciembre de 1521.
}

«De la laguna al océano: la presencia veneciana en el Golfo de Cádiz...» 
como correspondía a su función de cabecera final. Pero no fue así en todo momento: en los encantes inaugurales de 1436 el puerto sanluqueño era una parada opcional a discreción del patrón de la única galera prevista para ese año, con destino final en Cádiz y, opcionalmente, en Sevilla. Dos años más tarde Cádiz seguía siendo la última escala de la muda de Berbería, que además seguía compuesta por una única galera, la Querina. Así lo confirma Marino Sanudo en su entrada de agosto de $1438^{42}$.

Sanlúcar de Barrameda comenzó a contemplarse oficialmente como escala de la línea del Magreb a partir de la década de 1440. Así, en 1443 se previeron dos días en Cádiz y 24 días en Sanlúcar. Más tarde la escala gaditana desapareció de la ruta de Berbería, como revelan las órdenes de 1460. En 1462, de nuevo, solo figura Sanlúcar de Barrameda, con una parada prevista de 20 días $^{43}$. De esta manera se evitaba la competencia entre líneas, reservando Cádiz para la muda de Flandes y Sanlúcar para la de Berbería, aunque este fue el último año en que los convoyes para el Magreb central y occidental cruzaron el Estrecho de Gibraltar.

La escala de Sevilla es problemática. En el estudio de Bernard Doumerc sobre la muda de Berbería la capital hispalense solo figura en la transcripción de los encantes de la galera de 1436. Sin embargo, no es menos cierto que en 1442 los dos patrones de las galeras fondeadas en Sanlúcar se habían desplazado hasta la ciudad y enviaron una misiva al concejo en la que declaraban que la Serenísima estaba dispuesta a que los convoyes remontaran el Guadalquivir ${ }^{44}$. Con todo, a pesar de que el regimiento acordó conceder el seguro y salvoconducto que solicitaban, no hay constancia de que llegaran a hacerlo, al menos por el momento.

\footnotetext{
42 M. SANudo, Le vite dei Dogi (1423-1474), Venecia 1999, vol. 1, 188.

43 B. Doumerc, Venise et l'emirat hafside de Tunis (1231-1535), París 1999, 88-89, 230. 44 J.E. López de Coca Castañer, Las galeras..., 126-127 y doc. 1 del Apéndice.
} 
Aunque la flota de galeras estatales se ha hecho con el imaginario de la marina veneciana, hay que recordar que en Venecia también había navíos privados de menor tonelaje. Estas embarcaciones de titularidad privada estaban especialmente ligadas al comercio de la sal y del grano, cuyos circuitos no incluían la ruta de Poniente porque las naves hanseáticas entraban para aprovisionarse de sal hasta Ibiza, el punto de abastecimiento más occidental de los venecianos ${ }^{45}$. En consecuencia, no sorprende saber que estos navíos privados no fondeaban habitualmente en los puertos andaluces.

El número de embarcaciones de titularidad particular localizadas en el puerto gaditano es realmente reducido, menos de dos decenas para todo el siglo $\mathrm{XV}^{46}$. Algunas noticias proceden de viajes accidentados, como cuando la coca de Nicolò Rosso fue apresada en el puerto de Cádiz en 1403 por dos cocas genovesas, hecho recogido en el Codice Morosini ${ }^{47}$. Otro viaje accidentado es el que recoge el relato de los supervivientes del naufragio de la famosa nave Querina, que tras efectuar el viaje Creta-Cádiz-Canarias-Lisboa-Muros-Isla de Scilly terminó naufragando cerca del Círculo Polar Ártico en 1431. El patrón, Pietro Querini, anotó en su declaración sobre el naufragio que habían parado unos días en Cádiz desde el 3 de junio de ese año para reparar la embarcación ${ }^{48}$.

\footnotetext{
45 Sobre la navegación y el transporte de la sal en Venecia y el papel de la flota salinera en la marina de la república es de obligada consulta la obra clásica de J.-C. HOCQUET, Il sale e la fortuna di Venezia, Roma 1979, en especial 15-94 y 363-411.

46 Para un cuadro completo me remito a R. González Arévalo, Del Adriático al Atlántico...

47 Il Codice Morosini. Il mondo visto da Venezia (1094-1433), edizione critica, indici e altri apparati di A. NANETTI, Spoleto 2010, vol. 1, 215, 63.27 y 226, 63.48.

$48 \mathrm{El}$ relato de los supervivientes del naufragio ha sido editado y estudiado en $\mathrm{P}$. Querini, N. DE Michiele y C. Fioravante, Il naufragio della Querina. Veneziani nel circolo polare artico, a cura di P. NeLLI, postfazione di C. JUDDE DE LARIVIÈRE, Roma 2007. La declaración de Pietro Querini en la 47.
}

«De la laguna al océano: la presencia veneciana en el Golfo de Cádiz...» 
Alwyn Ruddock ya había observado que los mercaderes venecianos que se dirigían a Inglaterra empleaban fundamentalmente la muda de Flandes y prácticamente no llegaban carracas a Londres ${ }^{49}$. Pero, además, no he hallado rastro de los dos o tres navíos privados que, según Jacques Heers, solían acompañar al convoy de galeras de la república ${ }^{50}$.

En otras ocasiones las noticias llegan por vías diferentes: en febrero de 1456 los pilotos castellanos Juan de Bermeo y Álvaro del Río, enrolados en Cádiz para la travesía del Atlántico, reclamaban al patrón de la carraca, Loys Longue, que les pagara el salario acostumbrado por el trabajo desempeñado. Y en 1467 Domenico Malipiero recoge en sus Annales que se envió una galera desde Venecia hasta Sevilla para recuperar las mercancías del naufragio de otra nave que regresaba de Flandes.

\section{Nuevos datos sobre el comercio veneciano}

Los datos sobre el comercio veneciano en el Golfo de Cádiz se han centrado sobre todo en la actividad articulada a través de las galeras de la república ${ }^{51}$. Precisamente la presencia reducida de la navegación privada explica que no se le haya prestado prácticamente atención a su papel hasta la fecha, si bien los escasos datos localizados ayudan a completar el cuadro general conocido.

\footnotetext{
49 "Venetian merchants coming to England were firmly based on the capital and units of the Flanders Galleys unloaded cargoes there each year. Scarcely any Venetian carracks went to London, however". A. A. RudDOCK, Italian Merchants..., 55.

50 J. HeErs, Les génois en Anglaterre: la crise de 1458-1466, en Studi in onore di Armando Sapori, Milán 1957, vol. 1, 809. El insigne historiador francés citaba los datos recogidos por D. B. Quinn (ed.) y A. Ruddock (col.), The Port Books or Local Customs Accounts of Southampton for the Reign of Edward IV, Southampton 1937, vol. 2, "Table II, Genoese and Venetian carracks, 1461-1483", XLI-XLII. Sin embargo, la lectura de los apellidos de los patrones de las carracas revela que en su práctica totalidad eran genoveses, salvo Nicola Malipiero, lo que en realidad concuerda con lo afirmado por Ruddock, que ayudó a Quinn en la introducción histórica de su obra como directora de tesis doctoral. 51 J.E. LÓPEZ DE COCA CASTAÑER, Las galeras...
} 
La coca de Nicolò Rosso que fue apresada por otras dos cocas genovesas en el puerto de Cádiz llegaba un cargamento muy rico, valorado en más de 50.000 ducados de oro. La presa incluía especias y azúcar por valor de 40.000 ducados y algodón por 10.000 ducados y fue una represalia por la derrota de tres galeras genovesas en Modona, en el Peloponeso, antes de verse convertido en el gran centro distribuidor del Mediterráneo oriental gracias a la actividad veneciana ${ }^{52}$.

Los registros del consulado español de Brujas recogen que la carraca patroneada por Silvestre Polo, asaltada en 1455 por el genovés Giuliano Imperiale, transportaba un cargamento de 35 balas de arroz, 35 balas de almendras, 12 balas de dátiles, 15 jarras de orchilla, 3 jarras de aceite y 1 caja de confecciones, propiedad de Juan de Sevilla. Es decir, se trata no llevaba artículos de la tierra, además pertenecían a un mercader castellano, lo que añade nuevos datos sobre el papel de los venecianos en el Golfo de Cádiz y las opciones para el transporte de sus comerciantes ${ }^{53}$.

Las cuentas de Giovanni Foscari de la muda de Flades han dejado también algunos datos. Efectivamente, en el viaje de 146364, el noble veneciano vendió en Cádiz especias y paños, y adquirió atún, aceite, un puercoespín, un esclavo negro y otras mercancías no especificadas, además de cobrar el pasaje a dos individuos cuya identidad se desconoce. Las informaciones son algo más reducidas cuatro años más tarde. En 1467 Foscari compraba bizcocho, pan fresco y vino para la travesía; terciopelo de seda carmesí para hacerse un atuendo y varios esclavos musulmanes. Además, varios mercaderes que viajaban a bordo realizaron diversos fletes de mercancías desde Málaga a Cádiz a la

${ }^{52}$ Il Codice Morosini., vol. 1, p. 226, 63.48.

53 L. Gilliodts-van Severen (ed.), Cartulaire de l'Ancien Consulat d'Espagne à Bruges. Première partie de 1280 à 1550, Brujas 1901, 67, 26-VI-1467.

«De la laguna al océano: la presencia veneciana en el Golfo de Cádiz...» 
ida, y de Inglaterra a Cádiz, y de Cádiz a Málaga y a Orán a la vuelta $^{54}$.

Hay un dato interesante relacionado con la ermita de San Sebastián, en el islote rocoso del mismo nombre. La tradición sostiene que en 1457 la tripulación de una embarcación veneciana llegó enferma de peste a la ciudad. No se les permitió entrar en ella, pero sí instalarse en la isla de San Sebastián como lazareto. En testimonio de gratitud los venecianos habrían fundado allí una ermita y restauraron una atalaya musulmana que se había derrumbado. A pesar de la fecha de construcción indicada, José Sánchez Herrero recordaba que la primera cita documentada sobre la ermita es muy posterior, de un testamento otorgado en enero de $1514^{55}$. Ahora las cuentas de Giovanni Foscari confirman la existencia de la capilla ya la década posterior a la fecha tradicional de su fundación. Efectivamente, en marzo de 1467, durante la escala de Brujas, antes de partir de regreso hacia Venecia, Foscari adquirió un retablo para la ermita de San Sebastián, santo protector frente a la peste en 1457, por un voto que había realizado. El coste de la pieza ascendió a 8 ducados venecianos, lo que al cambio salió a 1.280 maravedíes ${ }^{56}$.

Otro dato curioso sobre la relación de Venecia con la ermita de San Sebastián de Cádiz lo revela Agustín de Horozco en su Historia de la ciudad de Cádir. (1598). En el "Capítulo Sexto: Punta de San Sebastián y la Torre que está en ella para atalaya y farol con que se guarda la costa", el cronista de la ciudad se hacía eco de que la torre había sido construida por los venecianos,

\footnotetext{
54 Las informaciones sobre el comercio en Cádiz en el viaje de 1463-64 en S. MonTEMEZZO, Giovanni Foscari..., 89, 97, 160-162, 168, 170, 183, 187; las operaciones del viaje de 1467-68 en 238, 240, 244, 318, 330, 345, 369, 372.

55 J. SÁnChez Herrero, Aspectos urbanísticos en Cádiz durante los siglos XIII al XV, en Cádiz en el siglo XIII. Actas de las "Jornadas Conmemorativas del VII Centenario de la muerte de Alfonso X el Sabio", Cádiz 1983, 33 (nota 72).

56 S. Montemezzo, Giovanni Foscari..., 318.
} 
como confirmaba un escudo de la república con el león de San $\operatorname{Marcos}^{57}$.

\section{El Golfo de Cádiz y Venecia: una relación para repensar}

A la luz de las informaciones reunidas y expuestas, la primera conclusión evidente es la necesidad de repensar la naturaleza y el alcance de la relación entre el Golfo de Cádiz y la República de Venecia en los últimos siglos medievales. El enorme peso de los intereses venecianos en el Mediterráneo oriental ha provocado, lógicamente, que la historiografía haya privilegiado su estudio frente a otras áreas de Europa occidental.

Al mismo tiempo, desde la perspectiva ibérica, o más específicamente andaluza, varios son los factores que hasta el momento no han permitido que se pudiera valorar adecuadamente el papel de los venecianos en Castilla, desde la escasez documental autóctona a la posición de privilegio de los genoveses.

La posición privilegiada del Golfo de Cádiz en la ruta de Poniente derivó en un conocimiento detallado de la costa, como revela la documentación náutica consultada, portulanos e informaciones sobre la navegación de las galeras venecianas. Para asegurar las mejores condiciones y defender los intereses de la república, la Serenísima no dudó en desplegar una diplomacia mercantil y abrir de un consulado en Cádiz, del que hay noticias en las décadas centrales del siglo XV y a principios del siglo XVI. La posición frente a Sanlúcar de Barrameda nunca estuvo en entredicho, a pesar de los contactos con los duques de MedinaSidonia.

57 A. DE Horozco, Historia de Cádiz, edición, introducción y notas a cargo de A. Morgado García, Cádiz 2001, 210.

«De la laguna al océano: la presencia veneciana en el Golfo de Cádiz...» 
Respecto al comercio de la marina de la república, nuevas fuentes como la contabilidad privada de Giovanni Foscari arrojan datos inéditos, de gran valor, sobre la realidad del comercio veneciano en Cádiz, adquiriendo productos de la zona, a la que exportaban tejidos tradicionales, particularmente de seda, y en la que se abastecían de vituallas para la travesía.

Las cuentas del noble veneciano confirman otro aspecto más de la relación de Venecia con Cádiz a través de la edificación de la ermita de San Sebastián en el islote del mismo nombre, en el que los venecianos también reconstruyeron la atalaya de origen musulmán y dejaron una piedra con las armas de San Marcos que representaban a la república. En definitiva, los intercambios entre la ciudad de la laguna, construida sobre más de un centenar de islas, y el Golfo de Cádiz, con el puerto sito en la isla del mismo nombre a la cabeza, se revelan cada vez más consistentes, a la espera de nuevos datos que permitan profundizar más aún en su naturaleza. 


\section{Bibliografía}

J. ABELLÁn PÉREZ, "Las barcas del Guadalete: las relaciones entre la villa de El Puerto de Santa María y la ciudad de Jerez de la Frontera en época de los Reyes Católicos", Revista de Historia de El Puerto, 47, (2011), 9-23.

Alcune raxion per marineri. Un manuale veneziano del secolo XV per gente di mare, O. PiTTARELlO (ed.), Venecia, 2006.

Calendar of State Papers Relating To English Affairs in the Archives of Venice, Volume 2, 1509-1519, R. BROWN (ed.), Londres, 1864.

M. BERTI, "Le aziende Da Colle: una finestra sulle relazioni commerciali tra la Toscana ed il Portogallo a metà del Quattrocento", en Toscana e Portogallo, "Miscellanea storica nel $650^{\circ}$ anniversario dello Studio Generale di Pisa", Pisa, 1994, 191-240.

A. DA CA' DA MOSTO, Portolano del mare, Venecia 1883.

A. DA MosTO, "Il portolano attribuito ad Alvise da Ca' da Mosto", Bollettino della Societa Geografica Italiana 3 (1893), 540-567.

L. D'Arienzo, "La presenza dei veneziani in Andalusia all'epoca di Cristoforo Colombo", en Atti del Convegno "Il letterato tramiti e realtà del Nuovo Mondo: Venezia, il mondo iberico e l'Italia”, Roma 1994, 203-230; reeditado en La presenza italiana in Spagna al tempo di Colombo, Roma 2010, 223-256.

B. Doumerc, Venise et l'emirat hafside de Tunis (1231-1535), París 1999.

P. FALCHETTA, "The Portolan of Michael of Rhodes", en P. O. LONG, D. MCGEe y A. M. STAHL (eds.), The Book of Michael of Rhodes. A Fifteenth-Century Maritime Manuscript, Cambridge (Mass.) 2009, vol. 3, 193-210.

L. Gilliodts-VAn Severen (ed.), Cartulaire de l'Ancien Consulat d'Espagne à Bruges. Première partie de 1280 à 1550, Brujas 1901.

J.D. GONZÁLEZ ARCE, "Pugnas entre monarquía y aristocracia por el control de los almojarifazgos costeros y otros derechos aduaneros de la Andalucía atlántica en el reino de los Reyes Católicos", Anuario de Estudios Medievales, 47/1 (2017), 73-106.

J.D. GONZÁlEZ ARCE, "La adaptación de la fiscalidad aduanera a los intereses repobladores, comerciales y políticos. Andalucía, 1241-1555”, Hispania, LXXVIII (2018), 39-67.

R. GonzÁlez ArÉvalo, La costa del Reino de Sevilla en la documentación náutica italiana (siglo XV), en A. MALPICA CUELLO, R.

«De la laguna al océano: la presencia veneciana en el Golfo de Cádiz...» 
G. Peinado Santaella, A. FÁbregas García (eds.), Historia de Andalucía. VII Coloquio, Granada, 2010, CD-ROM, 301-317.

R. GONZÁLEZ ARÉvAlO, Corso, comercio y navegación en el siglo XV: Castilla y las galeras mercantiles de Florencia, En la España Medieval, 34 (2011), 61-95.

R. GONZÁlez ARÉvalo, Presencia diferencial italiana en el sur de la Península Ibérica en la Baja Edad Media. Estado de la cuestión y propuestas de investigación, Medievalismo, 22 (2013), 175-208.

R. GonzÁlez ArÉvalo, Florentinos entre Cádiz y Sevilla en los siglos XIV y XV, en E. AZnAR VALLEJO, R. J. GONZÁlEZ ZALACAÍN (coords.): De mar a mar. Los puertos castellanos en la Edad Media, La Laguna, 2015, 273-308.

R. GONZÁLEZ ARÉVALO, Vecinos y propietarios: La integración de los italianos en las sociedades portuarias andaluzas (siglos XIII-XV), en J. Á. SolórZano TelecheA, B. AríZaga Bolumburu y M. BOCHACA (eds.), Las sociedades portuarias de la Europa atlántica en la Edad Media, Logroño, 2016, 249-284.

R. GONZÁlEZ ARÉVAlO, Integración y movilidad social de los italianos en la Corona de Castilla: genoveses, florentinos y venecianos en la Andalucía bajomedieval, L. TANZINI y S. TOGNETTI (eds.), Competenze, conoscenze e mobilità sociale nell'Italia del basso Medioevo, Roma, 2016, 375-401.

R. GonZÁlez ArÉvalo, Acordes y desacuerdos. Navegación y comercio de las galeras mercantiles de Venecia y Florencia en el Mediterráneo ibérico desde una perspectiva comparada, en R. GONZÁLEZ ARÉVALO (ed.), Navegación institucional y navegación privada en el Mediterráneo medieval, Granada, 2016, 145-191.

R. GONZÁlEZ ARÉVAlO, Presencias, interacciones y mutaciones italianas en el mar de Alborán (siglos XII-XV). Algunas reflexiones desde la historiografía, en B. Sarr (ed.), Alborán. Poblamiento e intercambios en las zonas costeras de al-Andalus y el Magreb, Granada, 2018, 361-410.

R. GONZÁlez ArÉvAlO, Del Guadalquivir al Nilo. El testamento de Andrea de Razi (1477) y la comunidad veneciana de Sevilla a finales del siglo XV, Nuova Rivista Storica, en prensa.

R. GonzÁlez Arévalo, Del Adriático al Atlántico: Venecia y Cádiz entre navegación, diplomacia y comercio (siglos XIV-XV), en prensa. R. GONZÁLEZ ARÉVALO, Cum necessarium sit habere consulem nostrum in Sibilia. El consulado veneciano de Sevilla (1402-1407), en prensa. 
L. GRECO (ed.), Quaderno di bordo di Giovanni Manzini, prete-notaio e cancelliere (1471-1484), Venecia, 1997.

J. HEERS, Les génois en Anglaterre: la crise de 1458-1466, en Studi in onore di Armando Sapori, Milán 1957, vol. 1, 809-832.

J.-C. HOCQUET, Il sale e la fortuna di Venezia, Roma, 1979.

A. DE Horozco, Historia de Cádiz, edición, introducción y notas a cargo de A. Morgado García, Cádiz, 2001.

Il Codice Morosini. Il mondo visto da Venezia (1094-1433), edizione critica, indici e altri apparati di A. NANETTI, Spoleto, 2010, 3 vols.

M.Á. LADERO QUeSADA, Unas cuentas en Cádiz (1485-1486), Cuadernos de Estudios Medievales II-III (1974-75), 85-120.

Libri commemoriali della Repubblica di Venezia. Regesti, Venecia 1901, tomo V.

P. O. Long, D. McGeE y A. M. STAHL (eds.), The Book of Michael of Rhodes. A Fifteenth-Century Maritime Manuscript, Cambridge (Mass.) 2009, 3 vols.

J.E. López DE Coca CASTAÑer, Las galeras venecianas de Poniente y Berbería desde la perspectiva española, Medievalismo, 16 (2006), 113-172. E. MARTín GutiÉRrez, Nuevos datos sobre la población y los genoveses en la ciudad de Cádiz. Una relectura, En la España Medieval, 29 (2006), 187-223.

L. MONGA (ed.), Un mercante di Milano in Europa. Diario di viaggio del primo Cinquecento, Milán 1985.

S. MontemeZzO, Giovanni Foscari. Viaggi di Fiandra 1463-1464 e $1467-$ 1468, Venecia 2012.

V. MuÑoz Gómez, Para el conocimiento de la costa de la Andalucía atlántica (siglos XIV-XVI): descripciones, relaciones y documentación náutica, Historia. Instituciones. Documentos 40, (2013) 179-205.

V. MuÑoz GÓmez, Puertos, abras, cabos e islas: la topografía medieval de la costa atlántica de Andalucía a través de las cartas portulanas (ss. XIV-XVI), en E. AZnAR VALlejO, R. J. GONZÁLEZ ZALACAÍN (coords.): De mar a mar. Los puertos castellanos en la Edad Media, La Laguna, 2015, 179-211.

V. MuÑoz GóMEZ, Derroteros, memoriales y saberes náuticos sobre las costas meridionales ibéricas durante la Era de los Descubrimientos (1526-c. 1555), Chronica Nova, 42 (2016), 425-451.

F. ORTALli (ed.), Lettere di Vincenzo Priuli, capitano delle galee di Fiandra, al doge di Veneria, 1521-1523, Venecia, 2005.

«De la laguna al océano: la presencia veneciana en el Golfo de Cádiz...» 
P. Querini, N. De Michiele y C. Fioravante, Il naufragio della Querina. Veneziani nel circolo polare artico, a cura di P. NELLI, postfazione di C. JUDDE DE LARIVIÈRE, Roma, 2007.

D. B. QUINN (ed.) y A. RUDDOCK (col.), The Port Books or Local Customs Accounts of Southampton for the Reign of Edward IV, Southampton, 1937, 2 vols.

A. RuDDOCK, Italian merchants and shipping in Southampton, 1270-1600, Southampton, 1951.

J. SÁnCHEZ Herrero, Aspectos urbanísticos en Cádiz durante los siglos XIII al XV, en Cádiz en el siglo XIII. Actas de las "Iornadas Conmemorativas del VII Centenario de la muerte de Alfonso X el Sabio”, Cádiz, 1983, 21-36.

M. SANUDO, Le vite dei Dogi (1423-1474), Venecia, 1999.

D. STÖCKLY, Le système de l'Incanto des galées du marché à Venise, fin XIIIe milieu XVe siècle, Boston-Leyden, 1995.

P. DE' VERSI, Raxion de' marineri. Taccuino nautico del XV secolo, A. CONTERIO (ed.), Venecia, 1991. 